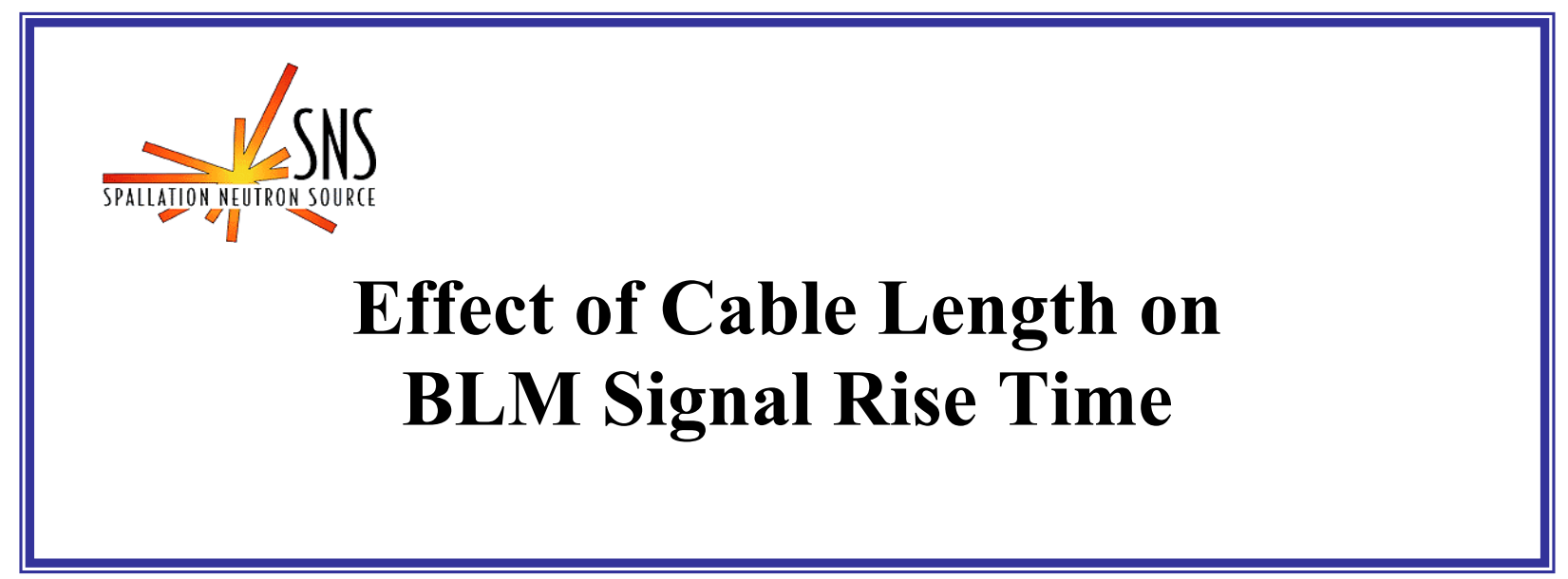

BNL/SNS TECHNICAL NOTE

NO. 130

\author{
R.L.Witkover and Mi Chaofeng
}

December 10, 2003 


\title{
Effect of Cable length on BLM Signal Rise Time
}

\author{
R. L. Witkover†, Mi Chaofeng* \\ $\dagger$ TechSource, Inc, Santa Fe, NM, * Brookhaven National Lab, Upton, NY
}

December 3, 2003

\section{Background}

An Ion Chamber signal behaves as a true current source until the voltage developed at the input of the monitoring circuit becomes a significant fraction of the bias voltage, which will never be the case for the SNS BLM. This unique characteristic has been exploited in the AFE circuit input stage design. As a result, the signal current does not depend on the load (opamp input resistor). Since the same current must flow through the opamp feedback resistor, the first stage output voltage is just the BLM signal current times the feedback resistance. Hence we are free to choose the value of the input resistor to satisfy other criteria.

There are opposing considerations in choosing the value of the input resistor. On the one hand, the voltage noise gain is given by the ratio of feedback to input resistors, favoring a large value of input resistance to minimize the amplification and increase the filtering of electrically coupled noise. On the other hand, the signal rise time, which is proportional to the input resistor and the distributed cable capacitance, will increase with larger input resistance. The requirement of a $10 \mu \mathrm{sec}$ output rise time led to the choice of $490 \mathrm{Ohms}$ as the input resistance. The cable capacitance is a factor in the input rise time, since for an un-matched load, the signal current must charge this capacitance.

Experience at BNL has determined that low tribo-electric cable ${ }^{1}$ must be used to measure nano-amp and lower signal currents. Low tribo-electric cables have been developed which reduce the noise generated by mechanical motion such as vibration from nearby fans, air ducts or water pipes. At BNL the cable which was successfully used was Belden 9054, a CATV cable with very low capacitance $(16.2 \mathrm{pF} / \mathrm{ft})$. This was discontinued by Belden and replaced by type 9224 with a capacitance of $22 \mathrm{pF} / \mathrm{ft}$, which results in an unacceptable rise time. Belden agreed to make a special run of 9054 for SNS. For a maximum cable run of 300 feet this would result in a $5 \mu \mathrm{sec}$ rise time.

The input amplifier's rise time is also affected by the opamp gain-bandwidth product and feedback roll-off. The signal from the first stage output is applied to the integrator channel and the fast and slow output channels. The slow output is not affected by signal rise time since it is heavily filtered and averaged over many pulses. The rise time of the fast output and integrator channels, however, are of interest and will be discussed below. The schematic is shown in Figure 1. 


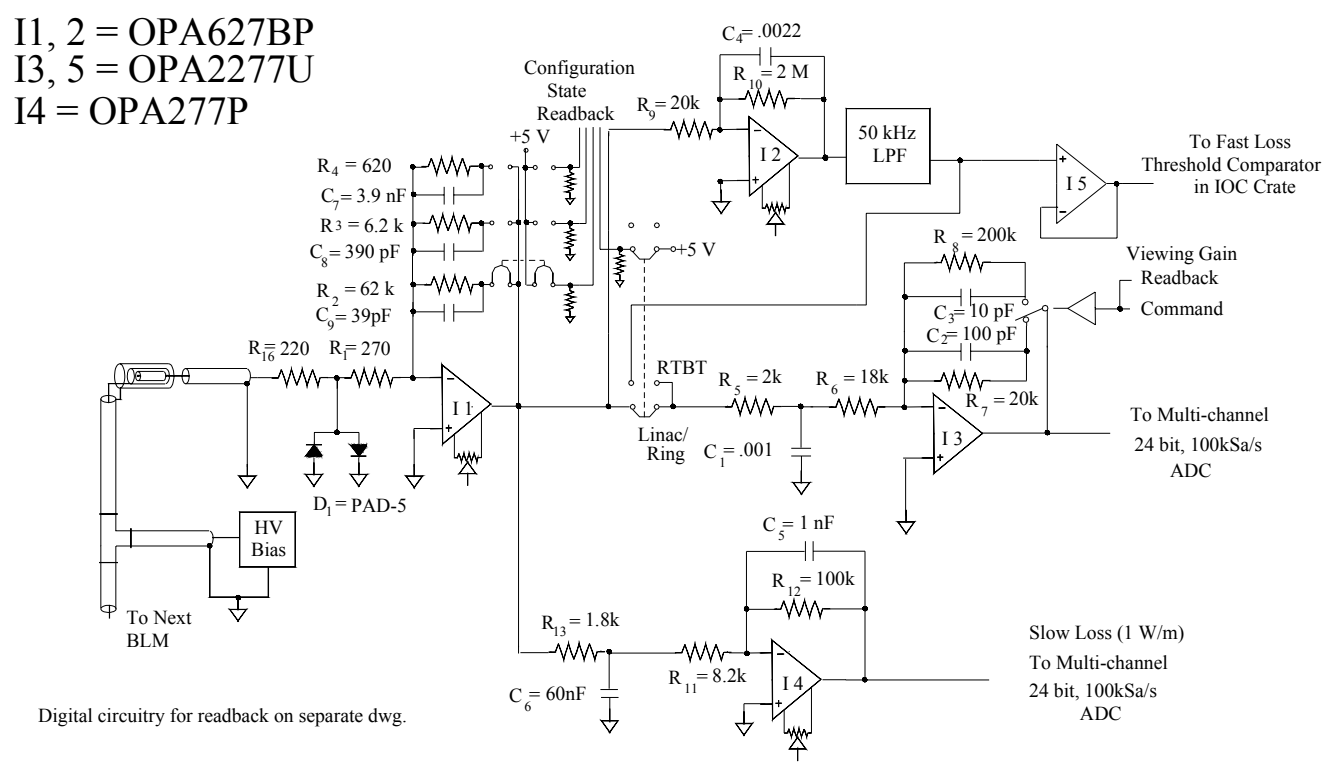

Figure 1. SNS BLM AFE Schematic

\section{The First Stage}

The first stage consists of a simple inverting opamp with the output rise time determined by the input RC roll-off, the closed loop bandwidth and the feedback roll-off. Slew rate is not a significant contribution since it is $55 \mathrm{~V} / \mu \mathrm{sec}$ for the OPA627BP, and will be ignored. The first stage output signal rise time will be given by:

Where:

$$
t_{r 1}=\sqrt{\left(2.2 * R_{\text {in }} * C_{\text {cable }}^{\prime} * D\right)^{2}+\left(2.2 * R_{f} * C_{f}\right)^{2}+\left(\frac{0.35}{B W_{1}}\right)^{2}}
$$

$$
\begin{array}{ll}
\mathrm{R}_{\text {in }}= & \text { Input load resistor }=490 \Omega \\
\mathrm{C}^{\prime} \text { cable } & \text { Cable capacitance } / \text { foot }=16.2 \mathrm{pF} / \mathrm{ft} \\
\mathrm{D}= & \text { Cable length in feet } \\
\mathrm{R}_{\mathrm{fl}}= & \text { Input stage feedback resistor }=6.2 \mathrm{~K} \Omega \\
\mathrm{C}_{\mathrm{fl}}= & \text { Input stage feedback capacitor }=390 \mathrm{pF} \\
\mathrm{BW}_{1}= & \text { Input stage opamp bandwidth }=16 \mathrm{MHz}
\end{array}
$$

The original design was based on a maximum cable length of 300 feet. Subsequently ${ }^{2}$ this was changed to a "typical" length of 420 feet and a maximum of 500 feet. The effect of cable length on the signal rise time out of the first stage is plotted in Figure 2. It is clear that the increased length does have a noticeable impact on the first stage output. The impact on the Fast Channel and Integrator channel outputs will also be estimated. 


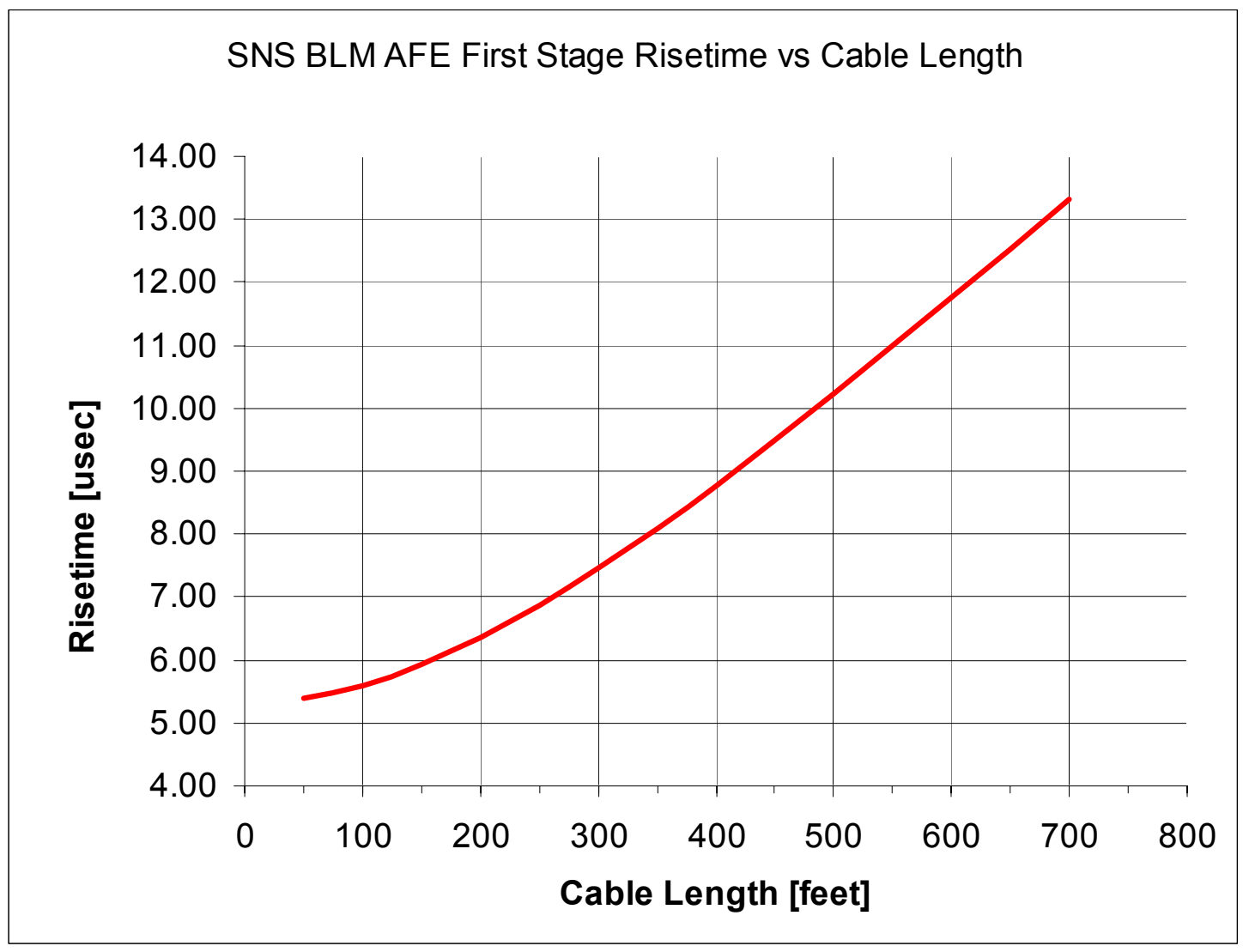

Figure 2. Effect of cable length on rise time of the first stage signal output.

\section{The Fast Channel}

Wideband linear data will be acquired from all BLM channels except those in RTBT, where the integral of the loss will be used. The first stage output will be applied to an inverting amplifier (The Viewing Gain stage) whose gain can be switched between 1 and 10 (see I3 in Figure 1). The Viewing Gain stage will further limit the rise time of the acquired data. In this case the output rise time will be given by:

$$
t_{\text {Fast }}=\sqrt{\left(t_{R 1}\right)^{2}+\left(2.2 * R_{f 2} * C_{f 2}\right)^{2}+\left(\frac{0.35}{B W_{2}}\right)^{2}}
$$

Where:

$\mathrm{t}_{\mathrm{R} 1}=\quad$ First stage rise time

$\mathrm{R}_{\mathrm{f} 2}=\quad$ Viewing Gain stage feedback resistor $=20 \mathrm{~K} \Omega$

$\mathrm{C}_{\mathrm{f} 2}=\quad$ Viewing Gain stage feedback capacitor $=100 \mathrm{pF}$

$\mathrm{BW}_{2}=\quad$ Viewing Gain stage bandwidth $=1 \mathrm{MHz}$ for $\mathrm{x} 1$ gain,

$0.1 \mathrm{MHz}$ for $\mathrm{x} 10$ gain 
The results are shown in Figure 3.

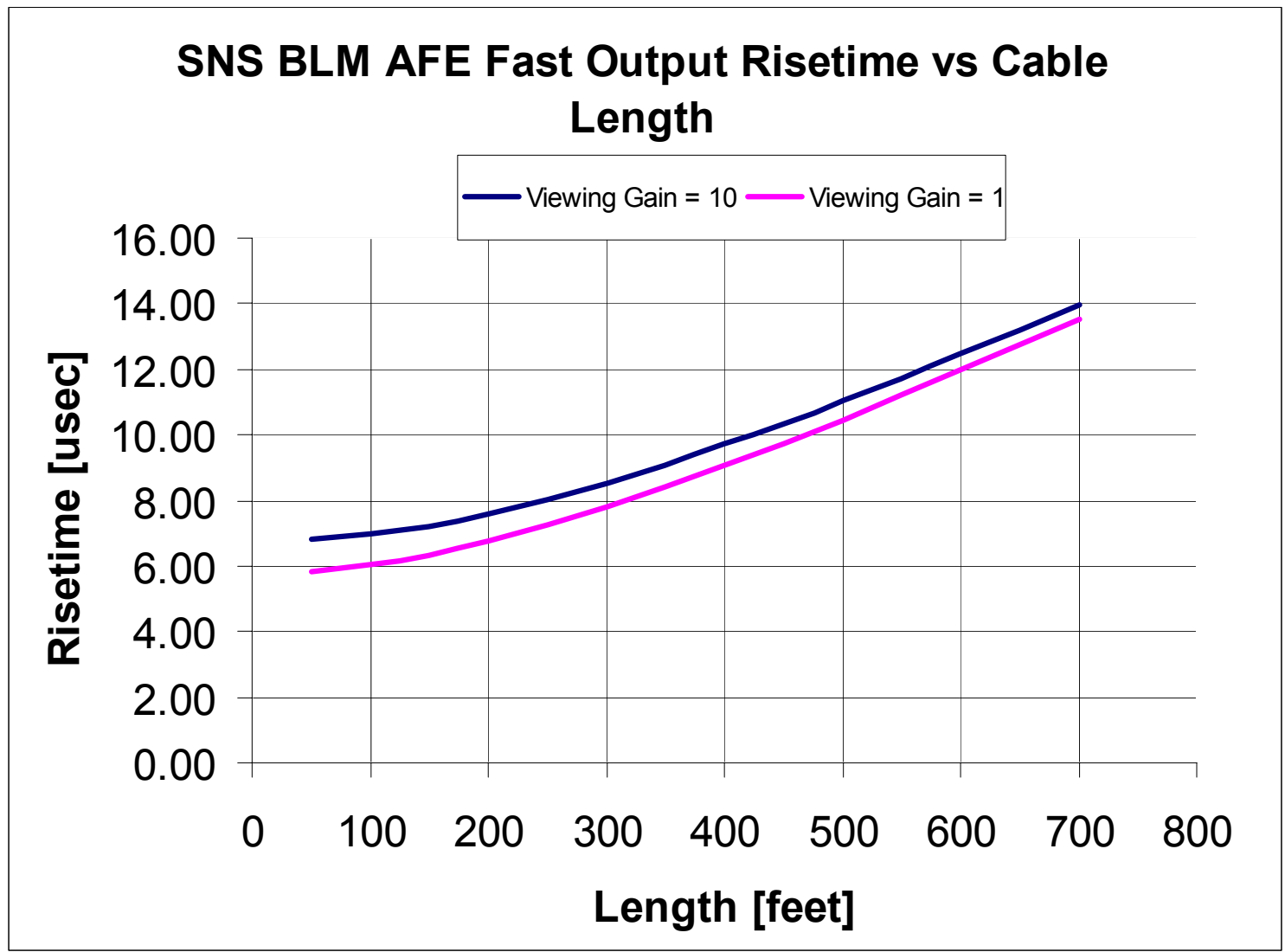

Figure 3. Effect of cable length on Fast Channel signal rise time for gains of 1 and 10.

It is clear that while the original 300 foot length would meet the design requirements the 500 foot length would be just over for the increased length of 500 feet. This is not a significant penalty.

\section{The Integrator Channel}

Besides data logging, the BLM system must provide a signal to the Machine Protect System (MPS) to inhibit the beam in the event of excessive loss. Early in the design, Mike Plum pointed out the advantages of a pulse-by-pulse integrated loss rather than linear loss signal to trip the MPS. To provide this function a "lossy Integrator" was added in the AFE design. In this circuit a large value resistor bleeds charge from the integrator capacitor, eliminating the need for gate and dump timing, making the circuit more reliable. However, some loss of signal linearity (about 5\%) results. The output of the first stage is applied to the integrator (I2 in Figure 1). As the losses continue the integrator output builds in amplitude and may become large enough to trip a comparator monitoring the signal. The effect of increased cable length on the Integrator Channel output has been estimated using a SPICE simulation which models actual circuit elements. Also included was the effect of the filter which follows the integrator. This low pass filter was added after excessive noise $(\sim 30 \mathrm{mV})$ was observed during tests at the 
BNL Linac with an early AFE prototype PC board. The integrator signal was sufficiently clean after passing it through a commercial (Krohn-Heit) filter. Subsequently an active filter was added to the circuit on the next PC layout revision.

Let us take the case of the most severe loss, that is, a $10 \mu \mathrm{sec}$ wide pulse that drives the first stage output to a near saturation level of 10 Volts. The finite rise time out of the first stage has been included. The effect on the integrator output for different cable lengths is shown in Figure 4.

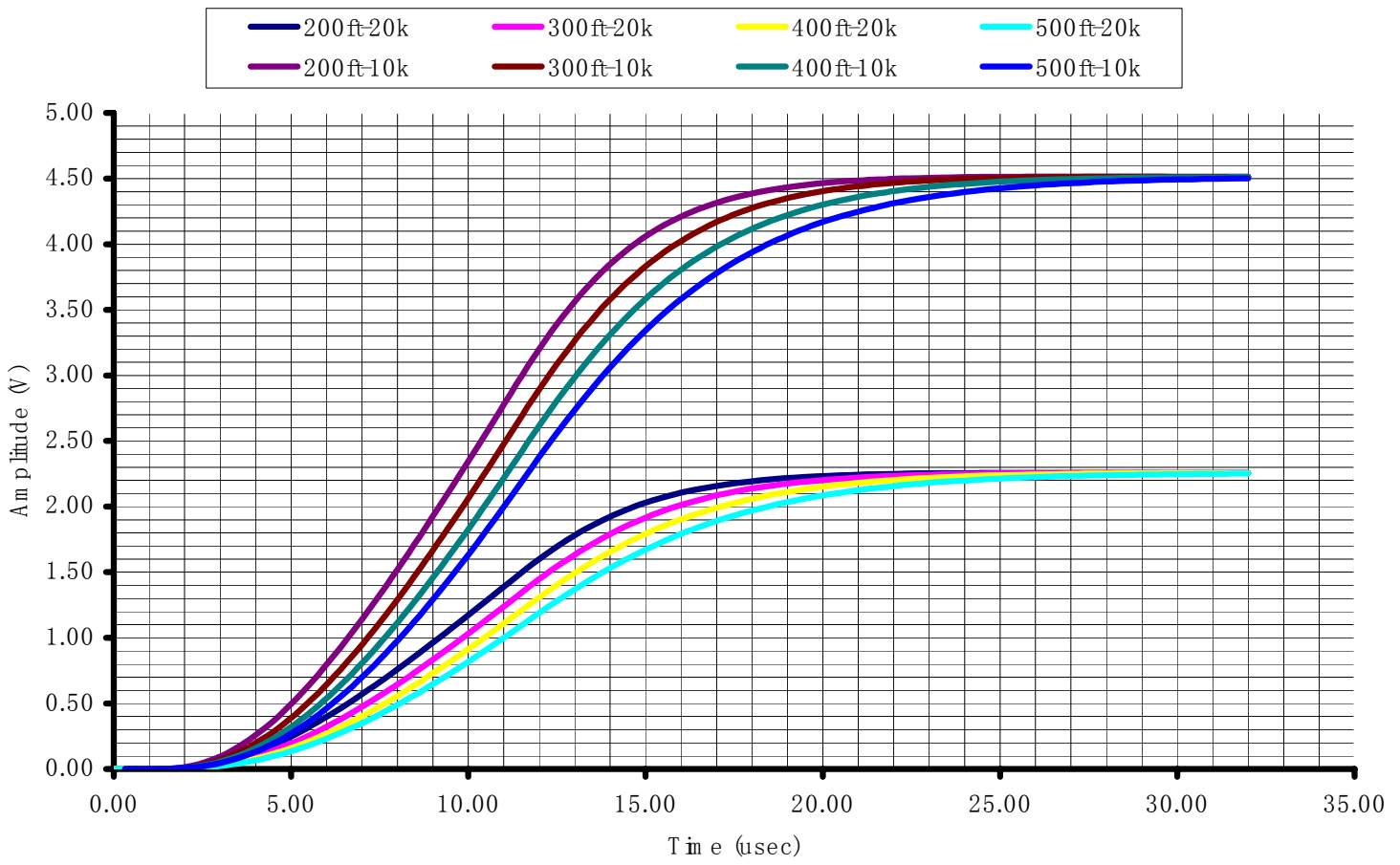

Figure 4. Integrator Signal output for various cable lengths for a 10 Volt first stage output. Values of the integrator input resistor of $10 \mathrm{k} \Omega$ and $20 \mathrm{k} \Omega$ are shown.

As can be seen from Figure 4, the effect of the finite first stage rise time appears as a delay of several microseconds, with the signal rising nearly linearly after that. Longer cable lengths contribute more to the delay, but the linear rise is almost parallel to that of the shorter cable lengths. Several things are clear from this simulation: (1) the effect of cable length variation from 200-500 feet is about $2 \mu \mathrm{sec}$, and, (2) a maximum loss over $10 \mu \mathrm{sec}$ will only produce a maximum of $2 \mathrm{~V}$ out with the current value of $20 \mathrm{k} \Omega$ integrator input resistor.

The "rise time" gets even worse when the effect of the low pass filter is included. (See Figure 5). The "delay" has now increased from 2 to $6 \mu \mathrm{sec}$ but the slope of the lines is essentially unchanged. This is unacceptable, however, the solution is available. It appears that the filter is no longer needed. Tests of all 8-channels of the present AFE PC board indicate that there is no difference in the noise before or after the filter. It is quite possible that the "noise" observed in the prototype PC board was due to an insipient oscillation and the final printed circuit layout, with additional board layers, has eliminated the problem, making the filter unnecessary. 
The concept of the MPS input rise time has been muddied since switching from the linear to integrated signal. Even an infinitely fast rising loss will produce only a linearly rising ramp out of the integrator. The finite rise time of the linear loss signal results in an effective delay of the start of the ramp. In any case, it is likely that the actual MPS reference levels will be set empirically, corresponding to tolerable (best achievable) loss levels which are gradually reduced as beam transport improves. Local specifics, such as distance from the loss and shielding due to intervening hardware and beam tuning requirements may cause different levels to be tolerated at various locations. In view of these considerations perhaps the MPS requirement on the BLM signal should be that it provides an output that will reach a reasonable level in $10 \mu \mathrm{sec}$.

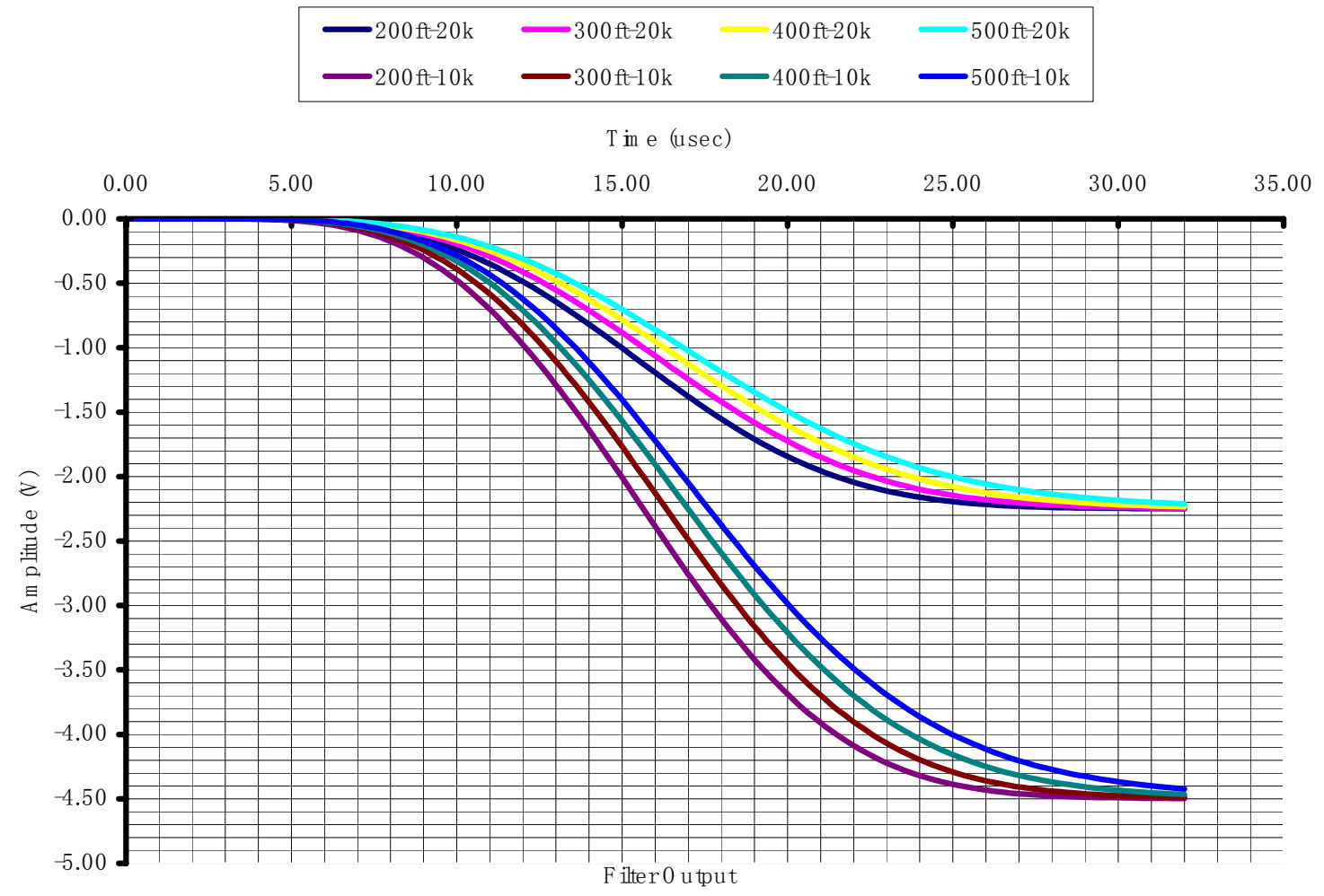

Figure 5. Output of the Low Pass Filter following the Integrator for various cable lengths and values of the integrator input resistor of $10 \mathrm{k} \Omega$ and $20 \mathrm{k} \Omega$.

\section{$\underline{\text { Recommendations }}$}

The effect of the increase in cable length from the original estimate of 300 feet maximum to 400 feet does cause an increase of several microseconds in the time before the MPS will trip, but it is a small effect. The increase of the linear loss signal rise time to 11-12 $\mu$ sec will have a minimal effect on the data logging since the ADCs sample at 100 $\mathrm{kSa} / \mathrm{sec}$. The real question is, in the face of a severe loss, will the signal to the MPS rise to a significant level in $10 \mu \mathrm{sec}$ to be detectable with a comparator? To achieve this the 
integrator gain must be increased by lowering the input resistor from $20 \mathrm{k} \Omega$ and $10 \mathrm{k} \Omega$, and the low pass filter following the integrator must be removed.

In the case of RTBT the cable length is unimportant. It may even be desirable to increase the input capacitance by adding lumped capacitors to the cable at the rack end. The reason is that in RTBT the losses occur in less than $1 \mu \mathrm{sec}$ rather than over the $1 \mathrm{msec}$ pulse, so a much smaller percentage loss will drive the first stage to saturation. There is no penalty to adding the capacitance in RTBT since we cannot see the structure of the fast losses with the ion chamber. The added capacitance will lower the peak input signal to reduce the possibility of input stage saturation, but will not change the total loss integral. It will, however, delay the time to reach the peak. This will not matter, since the beam is already out of the Ring and nothing can be done until the next pulse $16.667 \mathrm{msec}$ later.

${ }^{1}$ The Tribo-electric effect is familiar to anyone who has gotten a static electricity shock after walking on a rug on a low humidity day. It is the generation of a charge due to rubbing of insulating materials.

${ }^{2}$ J.D. Purcell. Email dated 9/29/03 